\title{
Occurrence and response to treatment of Graves' disease after COVID vaccination in two male patients
}

\author{
Luigi di Filippo $^{1} \cdot$ Laura Castellino $^{1} \cdot$ Andrea Giustina $_{(\mathbb{D}}^{1}$ \\ Received: 24 September 2021 / Accepted: 16 October 2021 / Published online: 2 November 2021 \\ (c) The Author(s), under exclusive licence to Springer Science+Business Media, LLC, part of Springer Nature 2021
}

COVID-19 had dramatic impact worldwide and various vaccines anti-SARS-CoV-2 were rapidly developed (https:// covid19.who.int/). Despite a good safety profile in clinical trials, rare and severe side effects of vaccines have been reported, including thyroid dysfunctions [1]. In particular, occurrence of Graves' disease (GD) post-vaccination with Tozinameran (Pfizer-BioNTech) was reported in three female subjects and only one male prevalently in young-adult age $[2,3]$ somewhat reflecting the epidemiological age and sex distribution of the disease [4]. Therefore, based on the previously reported cases a casual relationship between vaccination and occurrence of GD could not be excluded. Moreover, very little information is available on the clinical behavior of this possibly vaccine-related GD and potential occurrence of GD after COVID-19 vaccines other than Tozinameran. We here report the occurrence and the response to treatment of GD in two male patients after Vaxzevria (Oxford-AstraZeneca) SARS-CoV-2 vaccinations.

\section{Case 1}

A previously healthy, past-smoker, 32-year-old male patient, without any personal or family history of endocrine and autoimmune diseases, ten days after the second dose of Vaxzevria vaccine developed anxiety, tachycardia and palpitations, without signs of orbitopathy or local pain. These symptoms were not present after the first dose since the patient reported only general short-term self-limited mild side effects including pain and swelling at the injection site, fatigue and low-grade fever which resolved after few days. Thyroidfunction tests revealed decreased TSH with elevated free-T4

Andrea Giustina

giustina.andrea@hsr.it

1 Institute of Endocrine and Metabolic Sciences, San Raffaele VitaSalute University and IRCCS Hospital, Milan, Italy and free-T3 levels and elevated thyrotropin receptor-antibodies (TRAbs) (Table 1). Thyroid-ultrasonography revealed gland enlargement with pseudonodules and hypervascularization. He was initially treated with propranolol $40 \mathrm{mg} /$ daily and thiamazole $15 \mathrm{mg} /$ daily. However, he developed rush soon after starting thiamazole and was switched to propylthiouracil $150 \mathrm{mg} /$ daily. Biochemical and clinical response was good with progressive down-titration of treatment reaching rapidly euthyroidism. After three months of therapy his thyroid function is currently normal and TRAbs levels halved on $100 \mathrm{mg} /$ daily dose of propylthiouracil (Table 1 ).

\section{Case 2}

A previously healthy, past-smoker, 35-year-old male patient, without any personal or family history of endocrine and autoimmune disease, 5 days after the first dose of

Table 1 Biochemical data of two patients with Graves' disease diagnosis and after 3 months of treatment.

\begin{tabular}{lll}
\hline & Case 1 & Case 2 \\
\hline $\begin{array}{l}\text { TSH at diagnosis } \\
\text { TSH at } 3 \text { months of }\end{array}$ & $0.005 \mathrm{uIU} / \mathrm{mL}$ & $<0.004 \mathrm{uIU} / \mathrm{mL}$ \\
treatment & & $3.05 \mathrm{uIU} / \mathrm{mL}$ \\
fT3 at diagnosis & $7.9 \mathrm{pg} / \mathrm{mL}(2-4.4)$ & $\mathrm{missing}$ \\
fT3 at 3 months of & $3.06 \mathrm{pg} / \mathrm{mL}(2-4.4)$ & $3.3 \mathrm{pg} / \mathrm{mL}(2-4.4)$ \\
treatment & & \\
fT4 at diagnosis & $2.96 \mathrm{ng} / \mathrm{dL}$ & $4.96 \mathrm{ng} / \mathrm{dL}$ \\
& $(0.6-1.12)$ & $(0.6-1.12)$ \\
fT4 at 3 months of & $0.97 \mathrm{ng} / \mathrm{dL}$ & $0.69 \mathrm{ng} / \mathrm{dL}$ \\
treatment & $(0.6-1.12)$ & $(0.6-1.12)$ \\
TRAbs at diagnosis & $7.98 \mathrm{IU} / \mathrm{L}(<2.9)$ & $3.2 \mathrm{IU} / \mathrm{L}(<2.9)$ \\
TRAbs at 3 months of & $4.5 \mathrm{IU} / \mathrm{L}(<2.9)$ & $2.4 \mathrm{IU} / \mathrm{L}(<2.9)$ \\
treatment & &
\end{tabular}

TSH thyroid stimulating hormone, $f T 3$ free triiodothyronine, fT4 free thyroxine, TRAbs TSH Receptor Antibodies.

Reference ranges in parenthesis. 
Vaxzevria vaccine developed headache, nausea, asthenia, palpitations, tachycardia, mild eyes-redness and superior palpebral retraction. Thyroid-function tests revealed suppressed TSH with elevated free-T4 and free-T3 levels and elevated TRAbs (Table 1). Thyroid ultrasonography revealed gland enlargement and hypervascularization. He was treated with propranolol $20 \mathrm{mg} /$ daily and thiamazole $15 \mathrm{mg} /$ daily with a good clinical and biochemical response. After three months of therapy his thyroid function and TRAbs levels are currently normal on $5 \mathrm{mg} /$ daily dose of thiamazole (Table 1). Patient was counseled elsewhere not to undergo the second dose of the vaccine.

The necessary spread of vaccinations on a population basis may allow observation of their even rare side effects. Autoimmune endocrine diseases after vaccination have been previously reported possibly associated to autoimmune syndrome induced by adjuvants (ASIA) [5]. In genetically susceptible and predisposed subjects, particularly in those carrying specific HLA-DRB1 class II typing haplotypes or affected by previous autoimmune/inflammatory diseases and/ or allergic reactions, ASIA is triggered by several vaccine adjuvants and excipients leading to a dysfunctional immune response causing different pathological conditions and endocrinopathies $[5,6]$. Interestingly, our report adds to the current literature in that risk of inducing GD does not seem to be related to a unique mechanism. In fact, if GD after mRNA vaccine could be possibly related to its content in polyethylene glycol (PEG) lipids [2, 6], occurrence of GD after modified viral vaccine could be related to oil-in-water emulsion excipients such as polysorbate 80 [7, 8], since this latter vaccine does not contain PEG compounds.

To our knowledge, so far six cases including ours of GD after COVID-19 vaccination did occur [2, 3] and overall a 1:1 female male ratio has emerged. Therefore, our observation strengthens the hypothesis of a causal vaccine-GD relationship since males are rarely affected by GD and are known to be less genetically susceptible to develop autoimmune syndromes [2]. To date, no potential strong predictor or risk factor has yet been reported to influence GD occurrence after COVID-19 vaccination. Interestingly, both patients here reported were past-smokers, a factor widely known to affect immune and inflammatory response and autoimmune thyroid diseases occurrence including GD [9]. Moreover, hypovitaminosis D is widespread in Italy in both males and females [10] as well as in many parts of the world [11]. Interestingly, due to the known immunomodulatory role of Vitamin D (VD) [12], hypovitaminosis D has been reported to predispose both to COVID-19 occurrence and severity $[13,14]$ and to autoimmune thyroid disease [15]. Therefore, it cannot be excluded also a possible influence of VD status on GD occurrence after COVID-19 vaccination, although data on VD status in our patients were not available.
Furthermore, information on response to treatment of GD occurring after COVID-19 are scanty. In our two cases hyperthyroidism presented with relatively mildly elevated thyroid hormones and appeared to be well responding to medical treatment (either with thiamazole or propylthiouracil) with rapid restoration of euthyroidism and normalization or remarkable decrease of TRAbs with standard to low dose treatment [4].

In conclusion, although COVID-19 vaccination is currently not contraindicated in patients with stable autoimmune endocrine diseases $[16,17]$ our data suggest a note of caution in administering a further vaccine dose to patients that developed GD in the first weeks after vaccination. Moreover, we suggest to carefully consider if submitting patients with recent onset or non-stable GD to all types of COVID-19 vaccination.

Finally, it can be worth including also thyroid function monitoring in patients with marked and persistent pathological symptoms occurring after vaccination potentially overlapping with thyrotoxicosis such as fever, palpitations and asthenia particularly in those with personal or family history of autoimmune thyroid and non-thyroidal disease [18], since development of hyperthyroidism even not severe may have a negative impact on outcomes particularly in the geriatric population [19].

\section{Data availability}

All authors had full access to all the data in the study and take responsibility for the integrity of the data.

\section{Compliance with ethical standards}

Conflict of interest The authors declare no competing interests.

Publisher's note Springer Nature remains neutral with regard to jurisdictional claims in published maps and institutional affiliations.

\section{References}

1. B.G. İremli, S.N. Şendur, U. Ünlütürk. Three cases of subacute thyroiditis following SARS-CoV-2 vaccine: post-vaccination ASIA syndrome [published online ahead of print, 2021 May 27]. J. Clin. Endocrinol. Metab. 2021, dgab373. https://doi.org/10. 1210/clinem/dgab373

2. O. Vera-Lastra, A. Ordinola Navarro, M.P. Cruz Domiguez, G. Medina, T.I. Sánchez Valadez, L.J. Jara. Two cases of Graves' disease following SARS-CoV-2 vaccination: an autoimmune/ inflammatory syndrome induced by Adjuvants [published online ahead of print, 2021 May 3]. Thyroid. 2021; https://doi.org/10. 1089/thy.2021.0142

3. G. Zettinig, M. Krebs. Two further cases of Graves' disease following SARS-Cov-2 vaccination [published online ahead of print, 2021 Aug 3]. J. Endocrinol. Invest. 2021, 1-2. https://doi.org/10. 1007/s40618-021-01650-0 
4. A. Giustina, C. Ferrari, C. Bodini et al. Effects of methimazole treatment on growth hormone $(\mathrm{GH})$ response to GH-releasing hormone in patients with hyperthyroidism. Acta Endocrinol. (Copenh) 123(6), 613-618 (1990). https://doi.org/10.1530/acta.0.1230613

5. N.L. Bragazzi, A. Hejly, A. Watad, M. Adawi, H. Amital, Y. Shoenfeld, ASIA syndrome and endocrine autoimmune disorders. Best Pract. Res. Clin. Endocrinol. Metab. 34(1), 101412 (2020). https://doi.org/10.1016/j.beem.2020.101412

6. L.H. Garvey, S. Nasser, Anaphylaxis to the first COVID-19 vaccine: is polyethylene glycol (PEG) the culprit? Br. J. Anaesth. 126 (3), e106-e108 (2021). https://doi.org/10.1016/j.bja.2020.12.020

7. S. Shi, H. Zhu, X. Xia, Z. Liang, X. Ma, B. Sun, Vaccine adjuvants: understanding the structure and mechanism of adjuvanticity. Vaccine. 37(24), 3167-3178 (2019). https://doi.org/10.1016/ j.vaccine.2019.04.055. Epub 2019 Apr 29.

8. M. Greenhawt, E.M. Abrams, M. Shaker, et al. The risk of allergic reaction to SARS-CoV-2 vaccines and recommended evaluation and management: a systematic review, meta-analysis, GRADE assessment, and International Consensus Approach [published online ahead of print, 2021 Jun 18]. J. Allergy Clin. Immunol. Pract. 2021, S2213-2198(21)00671-1. https://doi.org/10.1016/j.ja ip. 2021.06 .006

9. A. Antonelli, S.M. Ferrari, F. Ragusa et al. Graves' disease: Epidemiology, genetic and environmental risk factors and viruses. Best Pract. Res. Clin. Endocrinol. Metab. 34(1), 101387 (2020). https://doi.org/10.1016/j.beem.2020.101387

10. M.L. Brandi, A. Giustina, Sexual dimorphism of coronavirus 19 morbidity and lethality. Trends Endocrinol. Metab. 31(12), 918-927 (2020). https://doi.org/10.1016/j.tem.2020.09.003

11. A. Giustina, R. Bouillon, N. Binkley et al. Controversies in vitamin D: a statement from the Third International Conference. JBMR Plus 4(12), e10417 (2020). https://doi.org/10.1002/jbm4. 10417. Published 2020 Nov 10
12. R. Bouillon, C. Marcocci, G. Carmeliet et al. Skeletal and extraskeletal actions of vitamin D: current evidence and outstanding questions. Endocr. Rev. 40(4), 1109-1151 (2019). https://doi.org/ 10.1210/er.2018-00126

13. J.P. Bilezikian, D. Bikle, M. Hewison et al. Mechanisms in endocrinology: vitamin D and COVID-19. Eur. J. Endocrinol. 183 (5), R133-R147 (2020). https://doi.org/10.1530/EJE-20-0665

14. di Filippo L., Allora A., Doga M., et al. Vitamin D levels associate with blood glucose and BMI in COVID-19 patients predicting disease severity [published online ahead of print, 2021 Aug 12]. J. Clin. Endocrinol. Metab. 2021, dgab599. https://doi.org/10.1210/ clinem/dgab599

15. D. Kim, The role of vitamin D in thyroid diseases. Int J Mol Sci. 18(9), 1949 (2017). https://doi.org/10.3390/ijms18091949. Published 2017 Sep 12

16. M. Puig-Domingo, M. Marazuela, B.O. Yildiz, A. Giustina, COVID-19 and endocrine and metabolic diseases. An updated statement from the European Society of Endocrinology. Endocrine 72(2), 301-316 (2021). https://doi.org/10.1007/ s12020-021-02734-w

17. A. Giustina, M. Marazuela, M. Reincke, B.O. Yildiz, M. PuigDomingo, One year of the pandemic - how European endocrinologists responded to the crisis: a statement from the European Society of Endocrinology. Eur. J. Endocrinol. 185(2), C1-C7 (2021). https://doi.org/10.1530/EJE-21-0397.

18. M. Gola, M. Doga, G. Mazziotti, S. Bonadonna, A. Giustina, Development of Graves' hyperthyroidism during the early phase of pregnancy in a patient with pre-existing and long-standing Hashimoto's hypothyroidism. J. Endocrinol. Invest. 29(3), 288-290 (2006). https://doi.org/10.1007/BF03345556

19. S. Bossoni, S. Cossi, A. Marengoni et al. The negative role of subclinical thyrotoxicosis on the outcome of hospitalized geriatric patients. J. Endocrinol. Invest. 25(10 Suppl), 64-65 (2002) 